\title{
Cognitive Rehabilitation of Dementia in Adults with Down Syndrome: A Review of Non-Pharmacological Interventions
}

\author{
Luciana Mascarenhas Fonseca $^{a}$ Anna Carolina Rufino Navatta ${ }^{c}$ \\ Cássio M.C. Bottino ${ }^{a} \quad$ Eliane Correa Miotto ${ }^{b}$ \\ ${ }^{a}$ Old Age Research Group, Department of Psychiatry and ${ }^{b}$ Department of Neurology, \\ University of São Paulo, and 'Department of Paediatrics, Federal University of São Paulo, \\ São Paulo, Brazil
}

\author{
Key Words \\ Dementia · Down syndrome $\cdot$ Rehabilitation · Therapeutics · Alzheimer's disease · \\ Early intervention $\cdot$ Geriatrics
}

\section{Abstract}

Background: There is a close genetic relationship between Alzheimer's disease (AD) and Down syndrome (DS), AD being the most severe mental disorder affecting ageing individuals with DS. The objective of the present study was to evaluate the efficacy of cognitive rehabilitation interventions in DS patients with AD by means of a critical literature review. Summary: Because $A D$ is progressive and irreversible, treatment is aimed at delaying and reducing the cognitive and functional decline in order to preserve or improve quality of life. The effects that pharmacological treatments and cognitive interventions have on elderly individuals with $A D$ are well documented. Recent clinical trials have investigated the use of pharmacological treatment in $\mathrm{DS}$ patients with $A D$, generating preliminary results that have been unfavourable. Key Messages: There is a clear lack of studies addressing the efficacy of cognitive rehabilitation interventions in $D S$ patients with $A D$, and there is an urgent need for studies providing evidence to inform decisions regarding the appropriate choice of treatment strategies.

(c) 2015 S. Karger AG, Basel

\section{Introduction}

Neuropsychiatric disorders are among the most severe and disabling age-related diseases [1]. Dementia is a neuropsychiatric disorder that affects cognition and function, having multiple aetiologies. Although the general concept of dementia remains the same and the 
Fonseca et al.: Cognitive Rehabilitation of Dementia in Adults with Down Syndrome: A Review of Non-Pharmacological Interventions

term itself is still used, the Diagnostic and Statistical Manual of Mental Disorders, fifth edition [2], employs a new term: major neurocognitive disorder. It is known that certain genetic and environmental characteristics can influence the development of dementia, the most prevalent form of which is Alzheimer's disease (AD).

The life expectancy of individuals with Down syndrome (DS) has increased considerably in recent years because of preventive health measures and advances in biomedicine. Nevertheless, atypical or premature ageing is a characteristic of individuals with DS, many of whom develop AD. Several studies have shown evidence of a genetic relationship between DS and AD. Trisomy 21 is associated with early and excessive deposition in the brain of beta-amyloid precursor protein, a protein that is associated with the neuropathological hallmarks of AD [3-5]. However, the diagnosis of dementia in individuals with DS remains challenging because of pre-existing cognitive deficits resulting from intellectual disability [6].

In an epidemiological study involving 506 individuals who had DS and were over 45 years of age, Coppus et al. [7] found the overall prevalence of dementia to be $16.8 \%$. The authors also demonstrated that the prevalence of dementia doubled every 5 years up to the age of 60 years, being $32.1 \%$ in individuals in the age bracket of 55-59 years and decreasing in those who were 60 years of age or older, probably due to the higher mortality rates among individuals with dementia. In a study involving 285 individuals who had DS and were in the age bracket of 35-74 years, the prevalence of dementia was found to be $13.3 \%$, the mean age at onset being 54.7 years [8]. In a study involving 646 adults with intellectual disability, the incidence of $\mathrm{AD}$ in individuals with DS was found to have increased from $4 \%$ in those who were 50 years of age to $67 \%$ in those who were 72 years of age [9]. Although not all adults with DS show clinical evidence of AD, all of those who are 40 years of age or older show neuropathological features consistent with $\mathrm{AD}$, including early formation of senile plaques and neurofibrillary tangles $[4,10]$. Therefore, dementia is the most serious problem faced by ageing individuals with DS [7].

Although AD can be treated pharmacologically or non-pharmacologically, a combination of pharmacological and non-pharmacological interventions increases treatment efficacy. Given that the disease is progressive and irreversible, the treatment of individuals with dementia is aimed at delaying or preventing cognitive decline.

One of the currently available non-pharmacological interventions is cognitive rehabilitation, which is aimed at optimising overall functioning, improving well-being, minimising losses, developing coping strategies, and avoiding harmful psychosocial processes [11]. Wilson [12] defined cognitive rehabilitation as a process in which patients, family members, and health professionals work together in order to improve cognitive difficulties following brain injury or neurological disease. In a recent review of cognitive training and cognitive rehabilitation for dementia, Bahar-Fuchs et al. [13] stated that the objective of cognitive rehabilitation is to tackle directly the cognitive difficulties considered most relevant by patients and their family members, as well as to target everyday challenges in the context of real-life situations that arise for the patients. Cognitive rehabilitation focussing on emotional and behavioural aspects is designated neuropsychological rehabilitation [14]. In 2011, the World Health Organization defined rehabilitation as a set of procedures and techniques aimed at helping disabled individuals restore or maintain an optimal level of functioning and interact with the environment [15]. Given the contrast between the meaning of the word rehabilitation (i.e., the act of restoring something to its original state) and the irreversibility of dementia, terms such as cognitive training, cognitive intervention, cognitive remediation, and cognitive stimulation are also used in the literature. Experimental studies have shown that cognitive rehabilitation is beneficial and that learning is possible for individuals during normal ageing [16] for those with mild cognitive impairment $[17,18]$ and for those with dementia [19-22]. 
Fonseca et al.: Cognitive Rehabilitation of Dementia in Adults with Down Syndrome:

A Review of Non-Pharmacological Interventions

Given the relationship between $\mathrm{AD}$ and $\mathrm{DS}$, as well as the peculiarities of $\mathrm{AD}$ and $\mathrm{DS}$ symptom development, it is extremely important to develop and evaluate the efficacy of interventions for individuals with DS and dementia. The pharmacological treatment used in individuals with dementia without DS, which includes drugs such as cholinesterase inhibitors and memantine, has recently been investigated for use in individuals with DS. Although the results are preliminary and have generally been unfavourable [23-26], further studies are currently underway. In addition to the fact that the benefits of pharmacological treatment in individuals with DS have yet to be confirmed, few studies have investigated non-pharmacological interventions for this population. In the present study, we sought to evaluate the efficacy of cognitive rehabilitation in DS patients with dementia by reviewing studies indexed in the PubMed and PsycINFO databases.

\section{Methods}

Given the lack of studies examining the efficacy of non-pharmacological interventions in DS patients with dementia, we chose not to limit our searches by the year of publication. We included case studies and broadened our search terms. We searched the PubMed and PsycINFO databases using the search terms 'rehabilitation', 'habilitation', 'intervention', 'training', 'cognitive stimulation', and 'remediation', all of which were always used in combination with the terms 'Down syndrome' and 'Alzheimer', as shown in table 1. The inclusion criteria were as follows: being a clinical trial or a case report, involving adults (i.e., individuals over 18 years of age) with DS, and including reports of cognitive rehabilitation interventions focussing on dementia.

\section{Results}

The searches performed and the articles retrieved are detailed in table 1 . A total of 12 searches were performed in the PubMed and PsycINFO databases collectively. Of the 150 articles initially selected, 6 met the criteria for inclusion in the present review, 5 being studies of interventions for individuals with DS (table 2) and 1 being a study of an intervention focussing on caregivers of individuals with DS (table 3). One study was initially considered for inclusion but was ultimately excluded because the participating DS patients had not been diagnosed with $\mathrm{AD}$; they were designated $\mathrm{DS} / \mathrm{AD}$ patients because they were over 35 years of age and were therefore assumed to have neuropathological features of AD [27]. In addition, a cognitive rehabilitation study involving a murine model of DS and AD was excluded because it did not address human interventions [28]. The remaining 142 studies were excluded because they did not address non-pharmacological interventions focussing on dementia in adults with DS.

Of the 150 articles initially selected, 59 (39\%) were review articles or editorials. Of those 59 articles, only 1 addressed the issue of pre-AD cognitive rehabilitation in DS patients, through the use of compensatory strategies [29], and none addressed the issue of cognitive rehabilitation in DS patients with dementia.

Vogl and Rapp [30] presented the case of a 52-year-old female who had DS and AD. The patient was a regular user of a recreational facility for the social inclusion of individuals with intellectual disability. The authors used a behavioural reinforcement intervention in order to reduce loitering and the theft of items belonging to other users of the facility. The authors performed an 11-day baseline evaluation and compared the results with those of an 11-day intervention consisting of differential reinforcement of other behaviour - which involves reinforcement of behaviours other than the target behaviour - and extinction of the inappro- 
Dementia

and Geriatric

Fonseca et al.: Cognitive Rehabilitation of Dementia in Adults with Down Syndrome: A Review of Non-Pharmacological Interventions

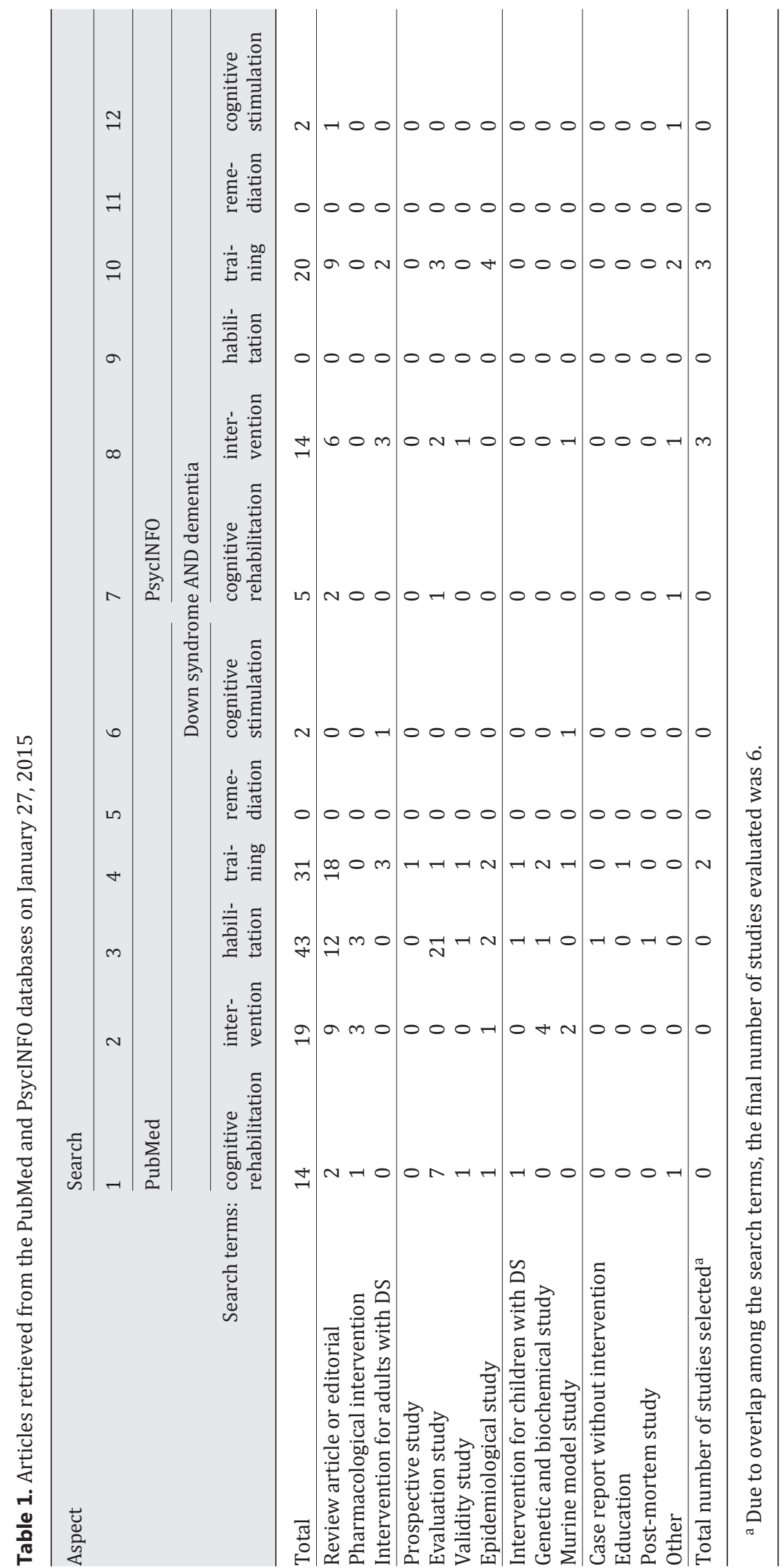




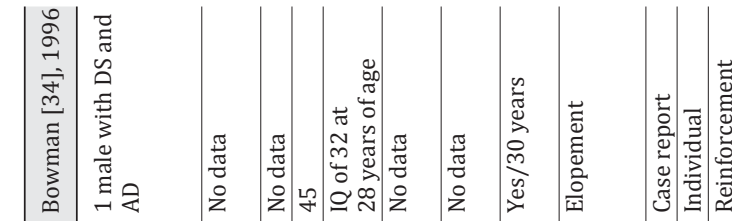
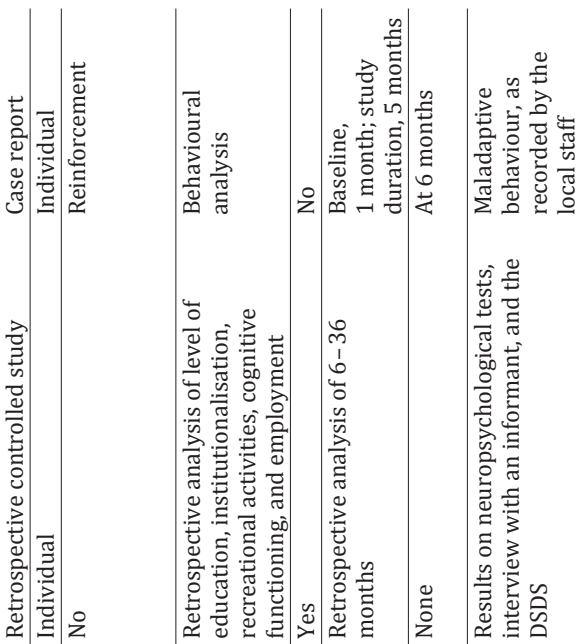

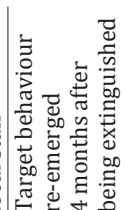

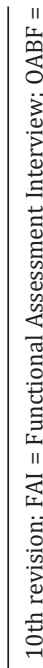
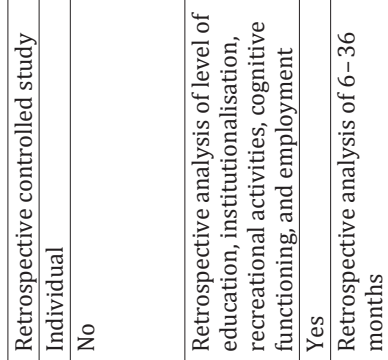

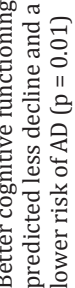

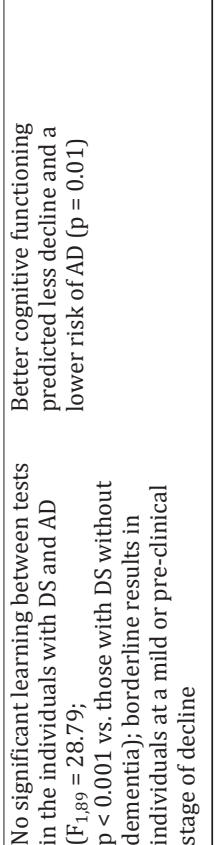

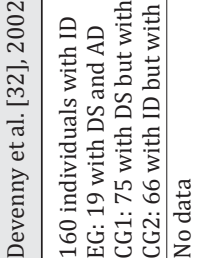
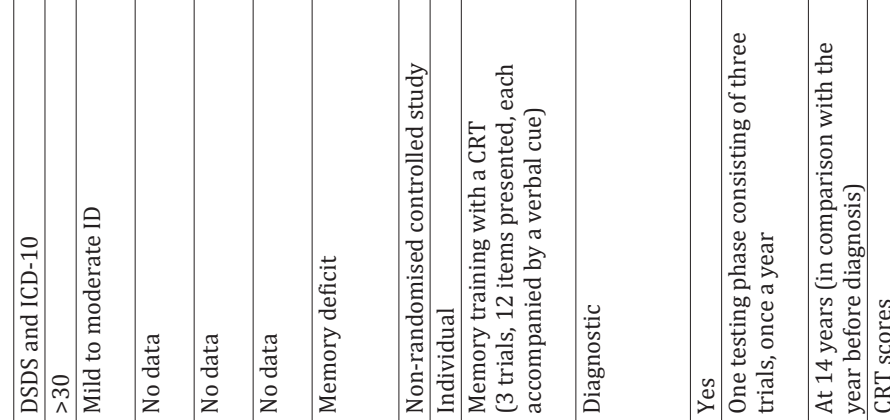

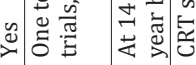
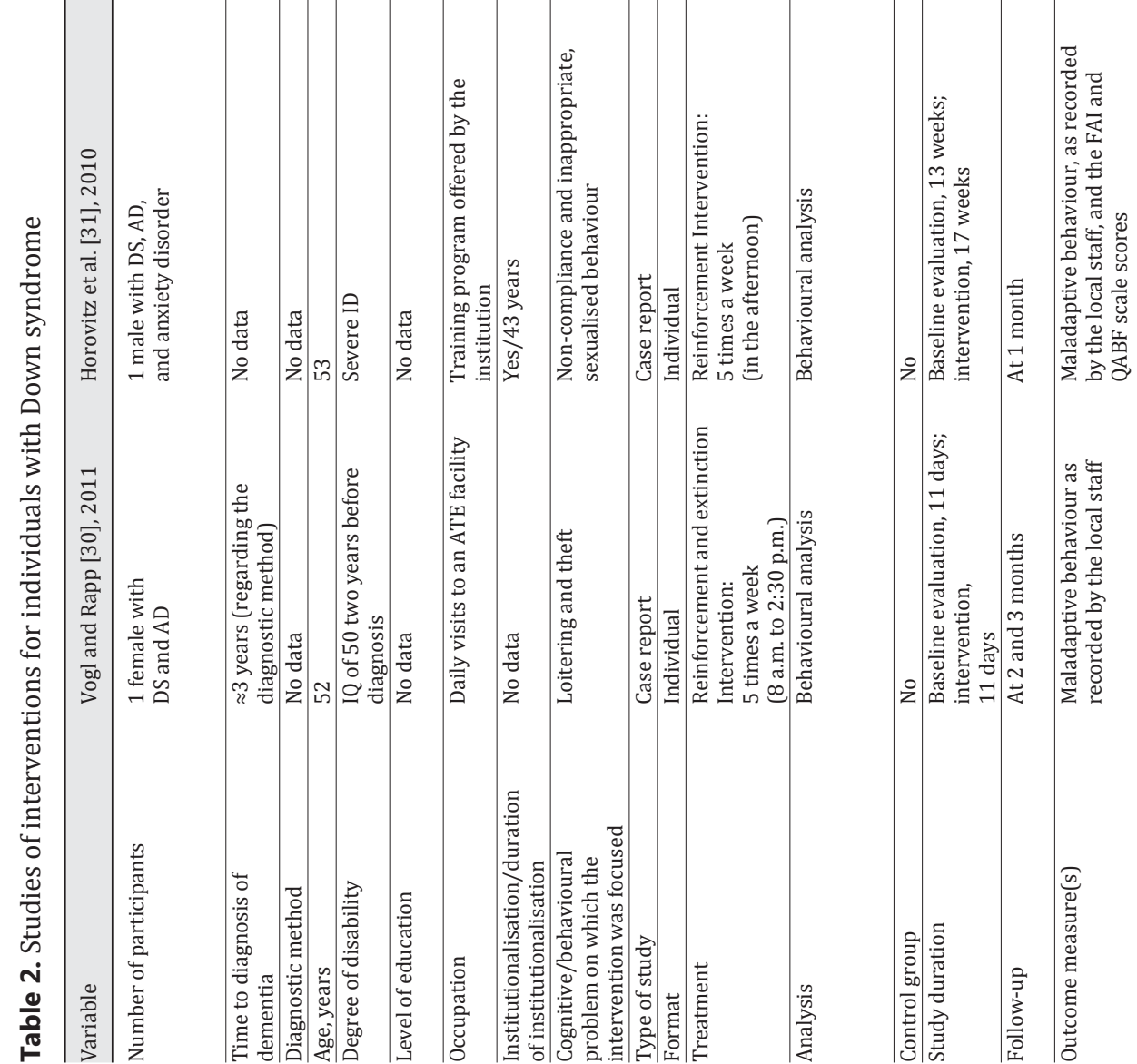

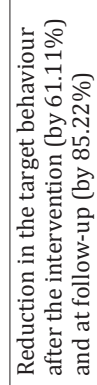
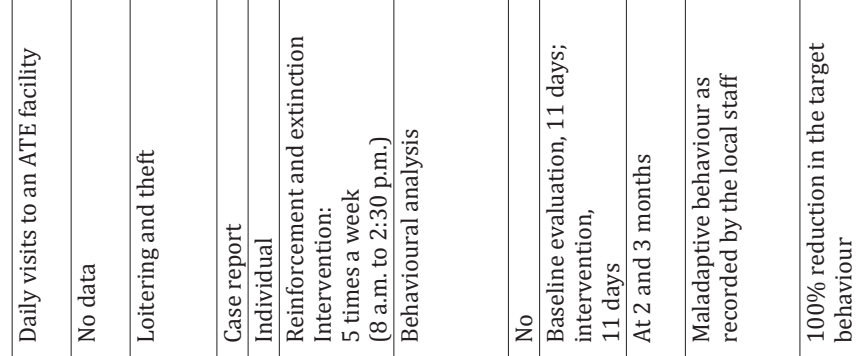
Table 3. Study of an intervention for caregivers of individuals with DS and AD

\begin{tabular}{|c|c|}
\hline Variable & Kalsy et al. [35], 2007 \\
\hline Caregivers & 97 \\
\hline Centres for DS and AD & 3 \\
\hline Duration of employment, months & $60.7 \pm 67.4$ \\
\hline Age, years & $42.2 \pm 10.63$ \\
\hline \multicolumn{2}{|l|}{ Gender } \\
\hline Male & $32(33)$ \\
\hline Female & $65(67)$ \\
\hline Level of education and appropriate training & No data \\
\hline Focus of the intervention & Education on ageing, dementia, and ID \\
\hline Type of study & Randomised uncontrolled study \\
\hline Format & Group intervention and individual analysis \\
\hline \multicolumn{2}{|l|}{ Cognitive/behavioural problem on } \\
\hline which the intervention was focussed & Loitering and theft \\
\hline Format & Individual \\
\hline Treatment & Psychoeducation with the use of 4 vignettes \\
\hline Control group & No \\
\hline Follow-up & None \\
\hline Outcome measures & $\begin{array}{l}\text { Controllability of Beliefs Scale score; knowledge of ageing } \\
\text { and ID; optimism question/mixed factorial design for } \\
\text { analysis of diagnosis of AD or no diagnosis; behavioural } \\
\text { excesses or deficits; and pre- or post-training }\end{array}$ \\
\hline Results & $\begin{array}{l}\text { Significant increase in knowledge after training } \\
\left(\mathrm{t}_{85}=4.1 ; \mathrm{p}<0.001\right) \text {, and training significantly decreased } \\
\text { the attribution of controllability }\left(\mathrm{F}_{2}=28.95 ; \mathrm{p}<0.001\right) .\end{array}$ \\
\hline
\end{tabular}

Values are means \pm SD or $\mathrm{n}(\%)$. ID = intellectual disability.

priate behaviour (theft), follow-up evaluations being performed at 2 and 3 months after the intervention. With the objective of reducing loitering, the patient was offered the possibility of manipulating coloured materials and engaging in art activities. The intervention targeting the problem of theft involved removing stolen items from patient lockers on a daily basis. The results showed that the intervention reduced loitering and theft, the follow-up evaluations at 2 and 3 months showing that reductions in both target behaviours were maintained over time. Methodological problems included the recording of data by the local staff and the fact that a detailed functional analysis was not performed, making it difficult to conclude that the change in behaviour was due to the intervention. Nevertheless, the fact that the target behaviour was extinguished suggests that the intervention was effective. The follow-up period was imposed because the patient required hospitalisation for dehydration. However, no functional measurements were performed before or after hospitalisation, which might have influenced the results.

Horovitz et al. [31] presented the case of a 53-year-old male patient with DS, AD, and anxiety disorder. The patient had been institutionalised since he was 10 years old and displayed inappropriate, sexualised behaviours. Before the intervention, the patient had been receiving reinforcement with verbal praise and attention, non-compliance resulting in the reinforcement being withheld. However, that strategy had not yielded favourable results. Compliance training and contingent reinforcement were used in order to reduce sexualised behaviours. Compliance with requests to allow assistance in getting dressed was reinforced 
by a combination of verbal praise, physical contact, and edible reinforcers. The interventions were performed every day of the week in the afternoon (after working hours of the patient), when the target behaviour occurred most frequently. The intervention was initially performed by two psychologists, subsequently by one psychologist and, finally, by a trained caregiver. The baseline evaluation lasted 13 weeks. The intervention lasted 17 weeks, the patient being subsequently followed for 4 weeks. The primary outcome measure was the occurrence of behavioural problems as recorded by the local staff. Non-compliance was found to have decreased by $61.11 \%$ after the intervention and by $85.22 \%$ at the end of follow-up. The study demonstrated the efficacy of contingent reinforcement in compliance training as well as the importance of carefully selecting a rewarding reinforcer. The authors concluded that it is possible to intervene to improve behaviour and compliance in individuals with DS and AD. The authors reported no data on patient literacy, patient level of education, time since diagnosis, or diagnostic methods.

Devenny et al. [32] conducted a study in which memory training was performed with the use of a cued recall test (CRT). However, rather than focussing on neuropsychological rehabilitation, the study focussed on the use of the CRT in the early diagnosis of memory deficits in adults with DS and mild to moderate intellectual disability. Nevertheless, the study showed relevant results regarding memory training with the CRT. The CRT performance of 19 adults with $\mathrm{DS}$ and $\mathrm{AD}$ was compared with that of two control groups: one comprising 75 adults with DS without dementia and the other comprising 66 adults with intellectual disability without DS. All adults were over 30 years of age and were participants in a 14-year follow-up study. The CRT consisted of a training period in which 12 items were presented, 4 at a time, each item being accompanied by a verbal category cue. Two measures were obtained: a free recall score (spontaneous recall of the list of 12 items); and a total score (free recall score plus items recalled when the category cue was provided). The authors concluded that the CRT can aid in the diagnosis of dementia and in the identification of a pre-diagnosis decline in memory function (sensitivity, 94.7\%; specificity, 93.9\%; positive predictive value, $81.9 \%$ ). With regard to rehabilitation, the study showed no significant learning between tests in the individuals with DS and AD, whose magnitude of learning was lower than that of individuals with DS without dementia $\left(\mathrm{F}_{1,89}=28.79 ; \mathrm{p}<0.001\right)$. Despite this finding, the authors concluded that individuals with early-stage dementia can learn by undergoing the CRT.

Temple et al. [33] performed a retrospective analysis of cognitive stimulation throughout the life of individuals with DS and the relationship between DS and AD, taking into consideration their level of education, employment, recreational activities, duration of institutionalisation, and level of cognitive functioning, all of which are considered to be protective factors in the general population. To that end, the authors analysed 35 adults with DS in the age bracket of 29-67 years. Multiple regression analyses revealed that a higher level of cognitive functioning predicted less decline and a lower risk of developing $A D(p=0.01)$. In addition, the level of cognitive functioning appeared to be associated with environmental factors such as level of education, years institutionalised, and employment $(\mathrm{p}=0.0001)$. This suggests that the relationship of these variables with symptoms is complex and indirect, calling for further investigation.

Bowman [34] presented the case of a 45-year-old male with DS and AD. The patient had been institutionalised for 30 years, and the intervention focussed on changing his elopement behaviour. The patient had attempted to elope on several previous occasions. On each occasion, he was taken back to the institution by car. Functional behavioural analysis revealed that the car rides and the attention given to the patient on those occasions reinforced his elopement behaviour, which was displayed again years later, when he developed the initial symptoms of dementia. The intervention consisted of eliminating the reinforcers of the undesirable behaviour and reinforcing the desired behaviour (i.e., staying in the common areas of 
Fonseca et al.: Cognitive Rehabilitation of Dementia in Adults with Down Syndrome: A Review of Non-Pharmacological Interventions

the institution). Elopement was reduced from 5 times a month at baseline to twice in the first month of intervention and only once in the second month. In the subsequent 3 months, he did not elope at all. However, the behaviour re-emerged in the following month. The author discussed the difficulties of and adjustments to the programme as a result of real-life limitations, having raised the hypothesis that dementia-related memory deficits interfered with the effects of the intervention after a few months.

Kalsy et al. [35] investigated the effects that psychoeducation regarding ageing, dementia, and intellectual disability had on 97 caregivers working with adults with DS and AD at three different centres. The mean age of the participants was 42.2 years, and the mean duration of employment was 60.7 months. The authors used a mixed factorial design in order to investigate three factors: diagnosis (dementia or no dementia), behaviour (deficits or excesses), and time (pre- vs. post-training). To that end, participants were randomly assigned one of four vignettes describing individuals with DS displaying behavioural deficits or excesses. Two vignettes described the person as having a diagnosis of $\mathrm{AD}$, whereas the other two suggested that further investigation was required in order to establish a diagnosis. Before and after training, participants completed the Controllability of Beliefs Scale [36] and a 20-item questionnaire on their knowledge of ageing and intellectual disability [37], as well as answering a question related to optimism regarding the potential for a change in the challenging behaviour [36]. There was a significant increase in knowledge after training $(p<0.001)$, and training significantly decreased the attribution of controllability $(p<0.001)$. The results suggest that training focussing on aspects of change relevant to behaviour can favourably influence caregiver knowledge and attributions of controllability within the context of individuals with DS and dementia.

None of the studies reviewed here included interventions that primarily focussed on cognitive rehabilitation for dementia. Three studies employed behavioural interventions [30, 31,34]; one study employed a memory training intervention with the use of a CRT to aid in the diagnosis of $\mathrm{AD}$ [32]; one study retrospectively analysed the role of cognitive stimulation throughout the life of individuals with DS as a protective factor for AD [33], and one study focussed on psychoeducational interventions for caregivers of DS patients with AD rather than for the patients themselves [35]. Common limitations of the studies reviewed here were as follows: being a case report or having a small sample size; having a non-randomised design; lack of generalisability of the results to the daily life of patients; lack of data on patient literacy; lack of investigation into the effects of interventions on activities of daily living, mood, behaviour, quality of life, stress, and well-being of caregivers; lack of use of the depression, anxiety, and stress scales; lack of investigation into emotional issues, and involving a short follow-up period or no follow-up.

\section{Discussion}

Our review of studies retrieved from the PubMed and PsycINFO databases and examining rehabilitation interventions for individuals with DS and dementia revealed an alarming lack of studies confirming the efficacy of such interventions. Although many studies have examined the efficacy of cognitive rehabilitation interventions for dementia patients in general $[17,18$, 20], few have examined the specificities of such interventions in individuals with DS presenting with evidence of pathological cognitive decline.

The heterogeneity of the studies precludes any detailed analysis of the current state of the art in non-pharmacological interventions. It is unclear whether individuals with DS and $\mathrm{AD}$ benefit from cognitive rehabilitation or other interventions. The few studies found in our review, the limitations of the relevant studies identified, and the fact that none focussed on 
cognitive rehabilitation allow us to state that the methodological rigour of a systematic review would have revealed an insufficient number of studies in this area, which has been neglected.

The studies reviewed here involved behavioural interventions, memory training as an evaluation measure, investigation of the protective role of cognitive stimulation throughout life, and psychoeducation for caregivers. The studies focussed primarily on behavioural analysis rehabilitation, possibly because changes in behaviour has been identified as an important feature that precedes or occurs concomitantly with cognitive decline in individuals with DS [38-40]. There have been few studies examining cognitive rehabilitation in DS patients with AD. Future studies should explore this issue, focussing on the efficacy of compensatory and restorative strategies, errorless learning, spaced retrieval, name-face association, visual imaging, use of cues, categorisation, hierarchical organisation, internal and external strategies, computerised training, functional magnetic resonance imaging, rehabilitation in combination with psychotherapy, rehabilitation involving family members, psychoeducation, generalisation to real life, and long-term maintenance of benefits. There is an urgent need for studies to support and justify the current therapeutic practices in this area.

Because of the high probability of AD in individuals with DS and the uncertainty regarding the efficacy of pharmacological treatments, cognitive rehabilitation interventions that focus on preventing and attenuating the development of $\mathrm{AD}$ and that can be performed at the first clinical signs of the disease (and even before the confirmation of neurodegeneration) are of great importance. It is known that there are a number of factors that increase cognitive reserve and can compensate for the effects of cognitive decline, reducing the clinical signs of AD in the general population; such factors include a healthy lifestyle, a challenging professional career, and adequate schooling [41-44]. However, it remains unclear whether the risk factors for AD in individuals with DS are the same as those for AD in the general population. In general, the aforementioned protective factors are compromised in individuals with intellectual disability, either because of the clinical features of the underlying disease or because of environmental factors (including social deprivation and low stimulation). The results of one of the studies included in the present review [33] confirm the hypothesis that, in individuals with intellectual disability, a higher level of cognitive functioning (developed throughout life) translates to less decline and a lower risk of developing AD. Therefore, one of the objectives of cognitive rehabilitation for individuals with intellectual disability without dementia is to bolster these protective factors by stimulating such individuals to fulfil their potential and by supporting their participation in the workforce, as well as by encouraging physical exercise, socialisation, personal satisfaction, and an active lifestyle. Future studies investigating the risk factors for AD in individuals with DS might clarify this relationship and indicate preventive treatments that are more effective. The fact that virtually all individuals with DS show neuropathological features of AD from the age of 30 onward [4] and the fact that such individuals have cholinergic deficits that are comparable to those found in the brains of individuals with AD [45] make individuals with DS a natural model of the neuropathological hallmarks of AD. Therefore, the development of interventions for this population can also be examined in terms of their applicability in and generalisability to elderly individuals with dementia.

The main limitation of the present study is the fact that we included only articles indexed in the PubMed and PsycINFO databases. Our literature review revealed that there is a large gap between research and practice regarding non-pharmacological interventions for DS patients with AD. This might be because cognitive rehabilitation has undergone major scientific breakthroughs in recent years and because the use of cognitive rehabilitation is becoming increasingly more common in specific areas. Nevertheless, it is impossible to explain the lack of studies evaluating the effects of currently used interventions in order to provide scientific evidence for their use and reproducibility. These interventions must be scientifically evaluated so that they can be disseminated and their quality can be controlled. 
Fonseca et al.: Cognitive Rehabilitation of Dementia in Adults with Down Syndrome: A Review of Non-Pharmacological Interventions

\section{Acknowledgments}

This study was supported by Fundação de Amparo a Pesquisa do Estado de São Paulo grant 2013/11571-9.

\section{Disclosure Statement}

None of the authors have any conflicts of interest.

\section{References}

1 Bottino CMC: Lessons from the epidemiology of neuropsychiatric disorders in older people. Arq Neuropsiquiatr 2012;70:165-166.

2 American Psychiatric Association: Diagnostic and Statistical Manual of Mental Disorders, ed 5. Arlington, American Psychiatric Association, 2013.

- 3 Kimura R, Kamino K, Yamamoto M, Nuripa A, Kida T, Kazui H, Hashimoto R, Tanaka T, Kudo T, Yamagata H, Tabara Y, Miki T, Akatsu H, Kosaka K, Funakoshi E, Nishitomi K, Sakaguchi G, Kato A, Hattori H, Uema T, Takeda M: The DYRK1A gene, encoded in chromosome 21 Down syndrome critical region, bridges between betaamyloid production and tau phosphorylation in Alzheimer disease. Hum Mol Genet 2007;16:15-23.

4 Wisniewski KE, Wisniewski HM, Wen GY: Occurrence of neuropathological changes and dementia of Alzheimer Disease in Down's syndrome. Ann Neurol 1985;17:278-282.

-5 Zigman WB: Atypical aging in Down syndrome. Dev Disabil Res Rev 2013;18:51-67.

-6 Silverman WP, Zigman WB, Krinsky-McHale SJ, Ryan R, Schupf N: Intellectual disability, mild cognitive impairment, and risk for dementia. J Policy Pract Intellect Disabil 2013;10:245-251.

7 Coppus A, Evenhuis H, Verberne GJ, Visser F, van Gool P, Eikelenboonm P, van Dujin C: Dementia and mortality in persons with Down syndrome. J Intellect Dis Res 2006;50:768-777.

-8 Tyrrell J, Cosgrave M, McCarron M, McPherson J, Calvert J, Kelly A, McLaughlin M, Gill M, Lawlor BA: Dementia in people with Down's syndrome. Int J Geriatr Psychiatry 2001;16:1168-1174.

-9 Zigman WB, Schupf N, Urv T, Zigman A, Silverman W: Incidence and temporal patterns of adaptive behaviour change in adults with mental retardation. Am J Ment Retard 2002;107:161-174.

10 Hof PR, Bouras C, Perl DP, Sparks DL, Mehta N, Morrison JH: Age-relate distribution of neuropathologic changes in cerebral cortex of patients with Down's syndrome. Arch Neurol 1995;52:379-391.

11 Clare L: Neuropsychological Rehabilitation and People with Dementia. New York, Psychology Press, 2008.

12 Wilson BA: Cognitive rehabilitation: how it is and how it might be. J Int Neuropsychol Soc 1997;3:487-496.

13 Bahar-Fuchs A, Clare L, Woods B: Cognitive training and cognitive rehabilitation for mild to moderate Alzheimer's disease and vascular dementia. Cochrane Database Syst Rev 2013;6:CD003260.

14 Wilson B: Evidence for the effectiveness of neuropsychological rehabilitation; in Wilson B, Gracey F, Evans JJ, Bateman A (eds): Neuropsychological Rehabilitation: Theory, Models, Therapy and Outcome. New York, Cambridge University Press, 2009, pp 22-36.

15 World Health Organization: World Bank World Report on Disability, chapt 4: Rehabilitation. Geneva, World Health Organization, 2011.

-16 Anderson ND, Guild EB, Cyr AA, Roberts J, Clare L: Contributions of frontal and medial temporal lobe function to the errorless learning advantage. Neuropsychol Rehabil 2012;22:169-186.

-17 Belleville S, Clément F, Mellah S, Gilbert B, Fontaine F, Gauthier S: Training-related brain plasticity in subjects at risk of developing Alzheimer's disease. Brain 2011;134:1623-1634.

18 Hampstead BM, Sathian K. Phillips PA, Amaraneni A, Delaune WR, Stringer AY: Mnemonic strategy training improves memory for object location associations in both healthy elderly and patients with amnestic mild cognitive impairment: a randomized, single-blind study. Neuropsychology 2012;26:385-399.

-19 Avila R, Carvalho IA, Bottino CM, Miotto EC: Neuropsychological rehabilitation in mild and moderate Alzheimer's disease patients. Behav Neurol 2007;18:225-233.

20 Dunn J, Clare L: Learning face-name associations in early-stage dementia: comparing the effects of errorless learning and effortful processing. Neuropsychol Rehabil 2007;17:735-754.

-21 Plaza V, López-Crespo G, Antúnez C, Fuentes LJ, Estévez AF: Improving delayed face recognition in Alzheimer's disease by differential outcomes. Neuropsychology 2012;26:483-489.

-22 Small JA: A new frontier in spaced retrieval memory training for persons with Alzheimer's disease. Neuropsychol Rehabil 2012;22:329-261.

23 Hanney M, Prasher V, Willians N, Jones EL, Aarsland D, Corbett A, Lawrence D, Yu LM, Tyrer S, Francis PT, Johnson T, Bullok R, Ballard C: Memantine for dementia in adults older than 40 years with Down's syndrome (MEADSOWS): a randomised, double-blind, placebo-controlled trial. Lancet 2012;379:528-536. 
Fonseca et al.: Cognitive Rehabilitation of Dementia in Adults with Down Syndrome: A Review of Non-Pharmacological Interventions

24 Lott IT, Doran E, Nguyen VQ, Tournay A, Head E, Gillen DL: Down syndrome and dementia: a randomized, controlled trial of antioxidant supplementation. Am J Med Genet 2011;155:1939-1948.

25 Prasher VP, Adams C, Holder R: Long term safety and efficacy of donezepil in the treatment of dementia in Alzheimer's disease in adults with Down syndrome: open label study. Int J Geriatr Psychiatry 2003;18:549551.

26 Mohan M, Carpenter PK, Bennett C: Donezepil for dementia in people with Down syndrome. Cochrane Database Syst Rev 2009;1:CD007178.

27 Woodruff-Pak DS, Romano S Papka M: training to criterion in eyeblink classical conditioning in Alzheimer's disease, Down syndrome with Alzheimer's disease, and healthy elderly. Behav Neurosci 1996;110:22-29.

28 Möhler H: Cognitive enhancement by pharmacological and behavioural interventions: the murine Down syndrome model. Biochem Pharmacol 2012;84:994-999.

29 Head E, Lott IT, Patterson D, Doran E, Haier RJ: Possible compensatory events in adult Down syndrome brain prior to the development of Alzheimer disease neuropathology: targets for nonpharmacological intervention. J Alzheimers Dis 2007;11:61-76.

-30 Vogl M, Rapp JT: Differential reinforcement of other behavior and extinction to reduce loitering and stealing for an adult with intellectual disability and dementia. Clin Case Stud 2011;10:229-235.

-31 Horovitz M, Kzlowski AM, Matson JL: Compliance training in an adult with dementia of the Alzheimer's type and Down syndrome. Clin Case Stud 2010;9:95-105.

-32 Devenny DA, Zimmerli EJ, Kittler P, Krinsky-McHale SJ: Cued recall in early-stage dementia in adults with Down's syndrome. J Intellect Dis Res 2002;46:472-483.

-33 Temple V, Jozsvai E, Konstantareas MM, Hewitt TA: Alzheimer dementia in Down's syndrome: the relevance of cognitive ability. J Intellect Dis Res 2001;45:47-55.

34 Bowman L: Behavior modification and Down syndrome dementia: a case study of treatment in a 'real life' setting. Dev Dis Bull 1996;24:58-67.

35 Kalsy S, Heath R, Adams D, Oliver C: Effects of training on controllability attributions of behavioural excess and deficits shown by adults with Down syndrome and dementia. J Appl Res Intellect Dis 2007;20:64-68.

-36 Dagnan D, Grant F, McDonnell A: Understanding challenging behaviour in older people; the development of the Controllability Beliefs Scale. Behav Cogn Psychother 2004;32:501-506.

37 McCallion PM, Janicki MP: Knowledge of Ageing and Intellectual Disabilities Quiz. Intellectual Disabilities and Dementia: Computer Based Course. Professional Development Programme. New York, Rockefeller College, University of Albany, 2002.

-38 Ball SL, Holland AJ, Watson PC, Huppert FA: Theoretical exploration of the neural bases of behavioural disinhibition, apathy and executive dysfunction in preclinical Alzheimer's disease in individuals with Down's syndrome: potential involvement of multiple frontal-subcortical neuronal circuits. J Intellect Disabil Res 2010; 54:320-336.

-39 Deb S, Hare M, Prior L. Symptoms of dementia among adults with Down's syndrome: a qualitative study. J Intellect Disabil Res 2007;51:726-739.

-40 Fonseca LM, Oliveira MC, Guilhoto LMFF, Cavalheiro EA, Bottino CMC: Bereavement and behavioral changes as risk factor for cognitive decline in adults with Down syndrome. Neuropsychiatr Dis Treat 2014;10:22092219.

41 Rosland S, Nordlund A, Eckerström C, Gustavsson MH, Zetterberg H, Wallin A: Biomarkers in relation to cognitive reserve in patients with mild cognitive impairment: proof of concept. Dement Geriatr Cogn Disord 2009;27:194-200.

42 Scarmeas N, Stern Y: Cognitive reserve and lifestyle. J Clin Exp Neuropsychol 2003;25:625-633.

-43 Snowdon DA, Greiner LH, Myarkesbery WR: Linguistic ability in early life and the neuropathology of Alzheimer's disease and cerebrovascular disease: findings from the Nun study. Ann NY Acad Sci 2000;903: 34-38.

44 Paillard-Borg S, Fratiglioni L, Winblad B, Wang H-X: Leisure Activities in Late Life in Relation to Dementia Risk: Principal Component Analysis. Dement Geriatr Cogn Disord 2009;28:136-144.

-45 Yates CM, Richie IM, Simpson J, Maloney AFJ, Gordon A: Noradrenaline in Alzheimer-type dementia and Down's syndrome. Lancet 1981;2:39-40. 